Article

\title{
Stability of Maximum Functional Equation and Some Properties of Groups
}

\author{
Muhammad Sarfraz (D), Qi Liu and Yongjin Li * \\ School of Mathematics, Sun Yat-Sen University, Guangzhou 510275, China; \\ sarfraz@mail2.sysu.edu.cn (M.S.); liuq325@mail2.sysu.edu.cn (Q.L.) \\ * Correspondence: stslyj@mail.sysu.edu.cn
}

Received: 4 November 2020; Accepted: 24 November 2020; Published: 26 November 2020

check for updates

\begin{abstract}
In this research paper, we deal with the problem of determining the function $\chi: G \rightarrow \mathbb{R}$, which is the solution to the maximum functional equation (MFE) $\max \left\{\chi(x y), \chi\left(x y^{-1}\right)\right\}=\chi(x) \chi(y)$, when the domain is a discretely normed abelian group or any arbitrary group $G$. We also analyse the stability of the maximum functional equation $\max \left\{\chi(x y), \chi\left(x y^{-1}\right)\right\}=\chi(x)+\chi(y)$ and its solutions for the function $\chi: G \rightarrow \mathbb{R}$, where $G$ be any group and also investigate the connection of the stability with commutators and free abelian group $K$ that can be embedded into a group $G$.
\end{abstract}

Keywords: maximum functional equations; discretely normed abelian group; stability of functional equation

MSC: 20D60; 54E35; 11T71

\section{Literature Review}

In [1], Volkmann proved that every function $\chi$ defined on an abelian group $\mathrm{G}$ can be described in the form $\chi(x)=|\alpha(x)|$, which is the solution of the maximum FE

$$
\max \left\{\chi(x y), \chi\left(x y^{-1}\right)\right\}=\chi(x)+\chi(y),
$$

where $\alpha: G \rightarrow \mathbb{R}$ is an additive function. Consequently, in [2], Toborg showed that Equation (1) also characterises the absolute value of additive functions when $G$ be any group. Their's main theorem is stated, as follows:

Theorem 1 (Toborg [2]). Let $G$ be any group ( $G$ is an abelian group (Volkmann [1])), then a function $\chi: G \rightarrow \mathbb{R}$ fulfills (1) if and only if there exists an additive function $\alpha: G \rightarrow \mathbb{R}$, such that $\chi(x)=|\alpha(x)|$ for any $x \in G$.

We recommend the readers to see [3,4], and the references cited therein in order to obtain comprehensive results related to functional Equation (1), which characterises the additive function's absolute value.

According to Simon and Volkmann [5], solutions of the MFE

$$
\max \left\{\chi(x y), \chi\left(x y^{-1}\right)\right\}=\chi(x) \chi(y)
$$

with the additional assumption that $\mathrm{G}$ is an additive abelian group was exhibited in the theorem, which is stated as: 
Theorem 2 ([5], Theorem 2). Assume that $G$ is an additive abelian group, whose each element is divisible by 6 (divisible by 2 and 3 ), and then a function $\chi: G \rightarrow \mathbb{R}$ fulfills

$$
\max \{\chi(x+y), \chi(x-y)\}=\chi(x) \chi(y)
$$

if and only if there exists an additive function $\alpha: G \rightarrow \mathbb{R}$ such that $\chi(x)=0$ or $\chi(x)=e^{|\alpha(x)|}$ for any $x \in G$.

Jarczyck and Volkmann [6] demonstrated the stability results of MFE (1) on additive abelian groups. Consequently, Badora et al. [7] also generalized their results in order to prove the stability for a certain class of groupoids. In [3], Gilanyi et al. determined the stability results of maximum functional equation $\max \{\chi((x \circ y) \circ y), \chi(x)\}=\chi(x \circ y)+\chi(y)$ on a square-symmetric groupoid. Consequently, they also examined the stability of maximum equation $\max \{\chi(x+y), \chi(x-y)\}=\chi(x)+\chi(y)$ on additive abelian groups. Various appropriate and useful results regarding stability can be found in papers by Przebieracz $[8,9]$.

This paper is arranged, as follows: in Section 2, we prove the functional Equation (1) for any arbitrary group $G$ without any characterisation of an additive function's absolute value. Besides, we also demonstrate some consistent and useful results concerning the normal subgroup of $G$.

In Section 3, we analyse the functional Equation (2) in order to obtain its solution. For this purpose, we drop the additional assumption that $G$ is an additive abelian group and is divisible by 6. We present the generalization of Theorem 2 by proposing a discretely normed abelian group $G$. Moreover, we investigate the functional Equation (2) for any arbitrary group $G$, which satisfies the Kannappan condition [10].

Section 4 deals with the stability results of the MFE (1) for a function $\chi: G \rightarrow \mathbb{R}$, where $G$ be any group and find some useful connection of stability with commutators and embedding of a group.

\section{Solutions of the Functional Equation (1)}

Throughout this article, let $G$ be any group, and 1 is considered to be the identity element of a group $G$.

Definition 1 ([10]). Let $G$ be an arbitrary group, we say a function $\chi: G \rightarrow \mathbb{R}$ satisfies the Kannappan condition if

$$
\chi(u v x)=\chi(u x v) \quad \text { for all } u, v, x \in G .
$$

Theorem 3. Let $G$ be a group and function $\chi: G \rightarrow \mathbb{R}$ satisfies the Kannappan condition then

$$
\max \left\{\chi(x y), \chi\left(x y^{-1}\right)\right\}=\chi(x)+\chi(y) \quad \text { for all } x, y \in G
$$

if and only if

$$
\min \left\{\chi(x y), \chi\left(x y^{-1}\right)\right\}=|\chi(x)-\chi(y)| \quad \text { for all } x, y \in G
$$

and also satisfies

$$
\chi\left(x^{2}\right)=2 \chi(x) \quad \text { for any } x \in G .
$$

Proof. Assume that $\chi$ satisfies Equation (3), and then by setting $x=y=1$ in (3), we can conclude that $\chi(1)=0$. It is also clear that $\chi(x) \geq 0$ and $\chi\left(x^{2}\right)=2 \chi(x)$ for any $x \in G$. The proof of Equation (4) consists of the following simple computation: 


$$
\begin{aligned}
& \max \left\{\chi(x y), \chi\left(x y^{-1}\right)\right\}+\min \left\{\chi(x y), \chi\left(x y^{-1}\right)\right\}=\chi(x y)+\chi\left(x y^{-1}\right) \\
& \chi(x)+\chi(y)+\min \left\{\chi(x y), \chi\left(x y^{-1}\right)\right\}=\chi(x y)+\chi\left(x y^{-1}\right) \\
& \chi(x)+\chi(y)+\min \left\{\chi(x y), \chi\left(x y^{-1}\right)\right\}=\max \left\{\chi\left(x y x y^{-1}\right), \chi\left(x y y x^{-1}\right)\right\} \\
& \chi(x)+\chi(y)+\min \left\{\chi(x y), \chi\left(x y^{-1}\right)\right\}=\max \left\{\chi\left(x^{2}\right), \chi\left(y^{2}\right)\right\} \\
& \min \left\{\chi(x y), \chi\left(x y^{-1}\right)\right\}=\max \{2 \chi(x), 2 \chi(y)\}-\chi(x)-\chi(y) \\
& \min \left\{\chi(x y), \chi\left(x y^{-1}\right)\right\}=|\chi(x)-\chi(y)| \text {. }
\end{aligned}
$$

Conversely, assume that Equations (4) and (5) hold. Subsequently, we can obtain

$$
\max \left\{\chi(x y), \chi\left(x y^{-1}\right)\right\}-\min \left\{\chi(x y), \chi\left(x y^{-1}\right)\right\}=\left|\chi(x y)-\chi\left(x y^{-1}\right)\right| .
$$

Using Equation (4) in the following computation, we get that

$$
\begin{aligned}
\max \left\{\chi(x y), \chi\left(x y^{-1}\right)\right\}-|\chi(x)-\chi(y)| & =\left|\chi(x y)-\chi\left(x y^{-1}\right)\right| \\
& =\min \left\{\chi\left(x y x y^{-1}\right), \chi\left(x y y x^{-1}\right)\right\} \\
& =\min \left\{\chi\left(x^{2}\right), \chi\left(y^{2}\right)\right\} \\
\max \left\{\chi(x y), \chi\left(x y^{-1}\right)\right\} & =2 \min \{\chi(x), \chi(y)\}+|\chi(x)-\chi(y)| \\
\max \left\{\chi(x y), \chi\left(x y^{-1}\right)\right\} & =\chi(x)+\chi(y) .
\end{aligned}
$$

Corollary 1. Let $G$ be a group and function $\chi: G \rightarrow \mathbb{R}$ satisfies the Kannappan condition, and then $\chi$ satisfies the Equation (3) if and only if

$$
\chi(x)+\chi(y)=\chi(x y)+\chi\left(x y^{-1}\right)-|\chi(x)-\chi(y)| \quad \text { for any } x, y \in G
$$

and also $\chi(1)=0$.

Proof. Suppose that $\chi(1)=0$ and Equation (6) holds. Subsequently, (6) can be written as

$$
2 \max \{\chi(x), \chi(y)\}=\chi(x y)+\chi\left(x y^{-1}\right) \quad \text { for any } x, y \in G .
$$

Because $\chi(1)=0$, then, for $x=y$, we can obtain that $\chi\left(x^{2}\right)=2 \chi(x)$. Afterwards, writing $x y$ instead of $x$ and $x y^{-1}$ instead of $y$ in (7), we can conclude that

$$
\begin{aligned}
2 \max \left\{\chi(x y), \chi\left(x y^{-1}\right)\right\} & =\chi\left(x y x y^{-1}\right)+\chi\left(x y^{2} x^{-1}\right) \\
& =\frac{1}{2}\left\{\chi\left(x^{2}\right)+\chi\left(y^{2}\right)\right\} \\
& =\frac{1}{2}\{2 \chi(x)+2 \chi(y)\} \\
\max \left\{\chi(x y), \chi\left(x y^{-1}\right)\right\} & =\chi(x)+\chi(y) .
\end{aligned}
$$

Conversely, assume that maximum Equation (3) holds, then, by Theorem 3, we can determine that $\chi(1)=0$ and Equation (6) holds.

Corollary 2. Let $G$ be a group and function $\chi: G \rightarrow \mathbb{R}$ satisfies the Kannappan condition, and then $\chi$ is a solution of equation

$$
\min \left\{\chi(x y), \chi\left(x y^{-1}\right)\right\}=|\chi(x)-\chi(y)| \quad \text { for any } x, y \in G
$$


and also satisfies

$$
\chi\left(x^{2}\right)=2 \chi(x) \quad \text { for any } x \in G
$$

if and only if there exists an additive function $\alpha: G \rightarrow \mathbb{R}$ such that $\chi(x)=|\alpha(x)|$ for any $x \in G$.

Theorem 4. Let $G$ be a group and function $\chi: G \rightarrow \mathbb{R}$ satisfies the Kannappan condition. If the Equations (4) and (5) are satisfied, then there exists a normal subgroup $N_{\chi}=\{v \in G \mid \chi(v)=0\}$ of $G$.

Proof. Because the function $\chi: G \rightarrow \mathbb{R}$ satisfies the Equations (4) and (5), then by Theorem 3

$$
\max \left\{\chi(x y), \chi\left(x y^{-1}\right)\right\}=\chi(x)+\chi(y) \quad \text { for any } x, y \in G .
$$

When we investigate Equations (3) and (4), then we can see that either $\chi(x y)=|\chi(x)-\chi(y)|$ then $\chi\left(x y^{-1}\right)=\chi(x)+\chi(y)$ or $\chi\left(x y^{-1}\right)=|\chi(x)-\chi(y)|$ then $\chi(x y)=\chi(x)+\chi(y)$. First, writing $v$ instead of $y$ and assume that $\chi(x) \neq 0$ for some $x \in G$ and also $\chi(x v)=\chi\left(x v^{-1}\right)$. Subsequently, we can compute that $\chi(x)+\chi(v)=|\chi(x)-\chi(v)|$. From Equation (3), we can easily deduce that $\chi(v), \chi(x) \geq 0$. Our assumption that $\chi(x) \neq 0$ forces $\chi(v)$ to be 0 .

From Equation (5), we can compute that $\chi(1)=0$, therefore $1 \in N_{\chi}$. Let $u, v \in N_{\chi}$. We can also deduce from Equation (3) that $\chi$ is even, so we have $\chi\left(v^{-1}\right)=\chi(v)=0$, therefore $v^{-1} \in N_{\chi}$. Additionally, $0=\chi(u)+\chi(v)=\max \left\{\chi(u v), \chi\left(u v^{-1}\right)\right\} \geq \chi(u v)$, but $\chi(x) \geq 0$ for all $x \in G$; therefore, we can obtain that $\chi(u v)=0$, so $u v \in N_{\chi}$. Hence $N_{\chi}$ is a subgroup of $G$. Let $v \in N_{\chi}$ and $x \in G$, then Equation (3) yields that

$$
\begin{aligned}
\chi(x)+\chi\left(x^{-1} v x\right) & =\max \left\{\chi\left(x x^{-1} v x\right), \chi\left(x x^{-1} v^{-1} x\right)\right\} \\
& =\max \left\{\chi(v x), \chi\left(v^{-1} x\right)\right\} \\
& =\max \left\{\chi\left(x^{-1} v^{-1}\right), \chi\left(x^{-1} v\right)\right\} \\
& =\chi\left(x^{-1}\right)+\chi(v) \\
\chi(x)+\chi\left(x^{-1} v x\right) & =\chi(x)+\chi(v) \\
\chi\left(x^{-1} v x\right) & =\chi(v)
\end{aligned}
$$

which implies that $\chi\left(x^{-1} v x\right)=\chi(v)$ for all $x \in G$ and $v \in N_{\chi}$. Hence, $N_{\chi}$ is a normal subgroup of $G$.

Corollary 3. For any group $G$, let a function $\chi$ on group $G$ satisfying Equation (3), then $\chi: G / N_{\chi} \rightarrow \mathbb{R}$ also satisfies

$$
\chi(x v)=\chi(x) \quad \text { for any } x \in G, v \in N_{\chi} .
$$

Additionally, $G / N_{\chi}$ is an abelian quotient group.

Proof. Let $x \in G$ be an arbitrary and assume that $v \in N_{\chi}$. We can deduce from (3)

$$
\begin{aligned}
\chi(x v) & \leq \max \left\{\chi(x v), \chi\left(x v^{-1}\right)\right\}=\chi(x)+\chi(v)=\chi(x) \\
& \leq \max \left\{\chi\left(x v^{2}\right), \chi(x)\right\}=\chi(x v)+\chi(v)=\chi(x v)
\end{aligned}
$$

that $\chi(x v)=\chi(x)+\chi(v)$. Hence, $\chi(x v)=\chi(x)$ for any $x \in G$ and $v \in N_{\chi}$. Moreover, we already proved that $\chi\left(x^{-1} v x\right)=\chi(v)$ for any $x \in G$ and $v \in N_{\chi}$, then, by replacing $v$ with $x v$, we can evaluate that $\chi(v x)=\chi(v x)$ for any $x \in G$ and $v \in N_{\chi}$. Additionally, $N_{\chi}$ is a normal subgroup of $G$; therefore, $G / N_{\chi}$ is an abelian quotient group. 
Corollary 4. Let $G$ be any group and function $\chi: G \rightarrow \mathbb{R}$ satisfy the Kannappan condition. If the maximum functional Equation (3) holds, then $\min \left\{\chi(x y), \chi\left(x y^{-1}\right)\right\} \in N_{\chi}$ for every $x, y \in G$.

Proof. Assume that $\chi$ satisfies the Equation (3) and Kannappan condition. Subsequently, from Theorem 3, we can compute that

$$
\min \left\{\chi(x y), \chi\left(x y^{-1}\right)\right\}=\chi(x)-\chi(y), \quad(\text { when } \chi(x) \geq \chi(y))
$$

or

$$
\min \left\{\chi(x y), \chi\left(x y^{-1}\right)\right\}=\chi(y)-\chi(x), \quad(\text { when } \chi(x)<\chi(y))
$$

combining both cases, we can see that $2 \min \left\{\chi(x y), \chi\left(x y^{-1}\right)\right\}=0$, therefore $\min \left\{\chi(x y), \chi\left(x y^{-1}\right)\right\}=$ 0 . In either case, it follows that $\min \left\{\chi(x y), \chi\left(x y^{-1}\right)\right\} \in N_{\chi}$ for every $x, y \in G$.

\section{Solutions of the Functional Equation (2)}

Theorem 5. Let $G$ be any arbitrary group, then a function $\chi: G \rightarrow \mathbb{R}$ satisfies

$$
\max \left\{\chi(x y), \chi\left(x y^{-1}\right)\right\}=\chi(x) \chi(y) \quad \text { for any } x, y \in G
$$

if and only if simultaneously $\chi(x)=0$ or

$$
\min \left\{\chi(x y), \chi\left(x y^{-1}\right)\right\}=\chi(x) \chi(y)^{-1} \vee\left[\chi(x) \chi(y)^{-1}\right]^{-1} \quad \text { for any } x, y \in G
$$

holds and satisfies

$$
\chi\left(x^{2}\right)=\chi(x)^{2} \quad \text { for all } x \in G .
$$

Proof. Suppose that $\chi$ satisfies Equation (9). Assume 1 as neutral element of group $G$, then by putting $x=y=1$ in (9), we can obtain that $\chi(1) \chi(1)=\chi(1)$, then $\chi(1)=0$ or $\chi(1)=1$. Let $\chi(1)=0$, then by Equation (9) we can compute $\chi(x)=0$ for any $x \in G$. Assume that $\chi(1)=1$, then from (9), It is easy to see that $\chi\left(x^{-1}\right)=\chi(x) \geq 0, \chi\left(x^{2}\right) \geq 1$ and $\chi\left(x^{2}\right)=\chi(x)^{2}$ for any $x \in G$. The proof of Equation (10) consists of the following simple computation:

$$
\begin{array}{rlrl}
\max \left\{\chi(x y), \chi\left(x y^{-1}\right)\right\} \cdot \min \left\{\chi(x y), \chi\left(x y^{-1}\right)\right\} & =\chi(x y) \chi\left(x y^{-1}\right) & \\
\chi(x) \chi(y) \cdot \min \left\{\chi(x y), \chi\left(x y^{-1}\right)\right\} & =\chi(x y) \chi\left(x y^{-1}\right) \\
\chi(x) \chi(y) \cdot \min \left\{\chi(x y), \chi\left(x y^{-1}\right)\right\} & =\max \left\{\chi\left(x y x y^{-1}\right), \chi\left(x y y x^{-1}\right)\right\} \\
\chi(x) \chi(y) \cdot \min \left\{\chi(x y), \chi\left(x y^{-1}\right)\right\} & =\max \left\{\chi\left(x^{2}\right), \chi\left(y^{2}\right)\right\} \\
\min \left\{\chi(x y), \chi\left(x y^{-1}\right)\right\} & =\max \left\{\chi(x)^{2}, \chi(y)^{2}\right\} \chi(x)^{-1} \chi(y)^{-1} \\
\min \left\{\chi(x y), \chi\left(x y^{-1}\right)\right\} & =\chi(x) \chi(y)^{-1} & (\text { when } \chi(x) \geq \chi(y)) \\
& \vee\left[\chi(x) \chi(y)^{-1}\right]^{-1} \quad & (\text { when } \chi(x)<\chi(y))
\end{array}
$$

Conversely, suppose that the Equations (10) and (11) are satisfied and $\chi(x) \neq 0$. Subsequently, it can be determined that

$$
\begin{aligned}
\max \left\{\chi(x y), \chi\left(x y^{-1}\right)\right\} \cdot \min \left\{\chi(x y), \chi\left(x y^{-1}\right)\right\}^{-1} & =\chi(x y) \chi\left(x y^{-1}\right)^{-1} \\
& \vee \chi(x y)^{-1} \chi\left(x y^{-1}\right) .
\end{aligned}
$$

Here, we have two cases, in the first case, using Equation (10) and (11), we derive the required result, as follows: 


$$
\begin{array}{rlrl}
\max \left\{\chi(x y), \chi\left(x y^{-1}\right)\right\} & =\chi(x y) \chi\left(x y^{-1}\right)^{-1} \cdot \min \left\{\chi(x y), \chi\left(x y^{-1}\right)\right\} & & \\
& =\chi(x y) \chi\left(x y^{-1}\right)^{-1} \chi(x) \chi(y)^{-1} & & (\text { when } \chi(x) \geq \chi(y)) \\
& =\min \left\{\chi\left(x y x y^{-1}\right), \chi\left(x y y x^{-1}\right)\right\} \chi(x) \chi(y)^{-1} & \\
& =\min \left\{\chi\left(x^{2}\right), \chi\left(y^{2}\right)\right\} \chi(x) \chi(y)^{-1} & \\
& =\min \left\{\chi(x)^{2}, \chi(y)^{2}\right\} \chi(x) \chi(y)^{-1} & \\
& =\chi(y)^{2} \chi(x) \chi(y)^{-1} & & (\text { when } \chi(x) \geq \chi(y)) \\
\max \left\{\chi(x y), \chi\left(x y^{-1}\right)\right\} & =\chi(x) \chi(y) . &
\end{array}
$$

From second case, when $\chi(x)<\chi(y)$, then we can also get that $\max \left\{\chi(x y), \chi\left(x y^{-1}\right)\right\}=$ $\chi(x) \chi(y)$.

Corollary 5. Let $G$ be a group and function $\chi: G \rightarrow \mathbb{R}$ satisfies the maximum Equation (9) if and only if

$$
\chi(x y) \chi\left(x y^{-1}\right)=\chi(x) \chi(y) \min \left\{\chi(x y), \chi\left(x y^{-1}\right)\right\} \quad \text { for any } x, y \in G
$$

and also $\chi(1)=1$.

Corollary 6. Let $G$ be a group and function $\chi: G \rightarrow \mathbb{R}$ is a solution of Equation (10) satisfies (11) if and only if there exists an additive function $\alpha: G \rightarrow \mathbb{R}$, such that $\chi(x)=e^{|\alpha(x)|}$ for any $x \in G$.

Definition 2 ([11]). Let $G$ be an abelian group. Subsequently, a function $\chi: G \rightarrow \mathbb{R}$ is said to be a discrete norm on $G$ if there exists some $\alpha>0$ such that $\chi(x)>\alpha$ whenever $x$ is not identity element of $G$. Afterwards, $(G, \chi)$ is called the discretely normed abelian group [11].

Simon and Volkmann [5] proved Theorem 2 with additional assumption that $G$ is an additive abelian group and is divisible by 6 , but we present the generalization of Theorem 2 by introducing the notion of discretely normed abelian group $G$, as follows:

Theorem 6. Assume that $(G, \chi)$ be a discretely normed abelian group, then a function $\chi: G \rightarrow \mathbb{R}$ fulfills (9) if and only if there exists an additive function $\alpha: G \rightarrow \mathbb{R}$, such that $\chi(x)=e^{|\alpha(x)|}$ for any $x \in G \backslash\{1\}$.

Proof. Because $G$ is a discretely normed abelian group, therefore there exists a discrete norm function $\chi: G \rightarrow \mathbb{R}$ such that $\chi(x)>0$ whenever $x \in G \backslash\{1\}$. Assume that $\eta(x)=\log \chi(x)$, then applying the main theorem of Toborg [2], a function $\chi: G \rightarrow \mathbb{R}$ satisfies

$$
\max \left\{\eta(x y), \eta\left(x y^{-1}\right)\right\}=\eta(x)+\eta(y)
$$

if and only if there exist an additive function $\alpha: G \rightarrow \mathbb{R}$ such that $\eta(x)=|\alpha(x)|$. Subsequently, we have

$$
\max \left\{\log \chi(x y), \log \chi\left(x y^{-1}\right)\right\}=\log \chi(x)+\log \chi(y)
$$

if and only if $\log \chi(x)=|\alpha(x)|$, which implies that

$$
\log \max \left\{\chi(x y), \chi\left(x y^{-1}\right)\right\}=\log \chi(x) \chi(y),
$$

thus, we conclude that

$$
\max \left\{\chi(x y), \chi\left(x y^{-1}\right)\right\}=\chi(x) \chi(y)
$$

if and only if there exists an additive function $\alpha: G \rightarrow \mathbb{R}$, such that $\chi(x)=e^{|\alpha(x)|}$ for any $x \in$ $G \backslash\{1\}$. 
Corollary 7. Let $(G, \chi)$ be a discretely normed abelian group, then a function $\chi$ is a solution of Equation (10) satisfying (11) if and only if there exists an additive function $\alpha: G \rightarrow \mathbb{R}$, such that $\chi(x)=e^{|\alpha(x)|}$ for any non-identity element $x \in G$.

Proof. From Theorem 6, we concluded that maximum functional Equation (9) holds; therefore, using Theorem 5 , we can also obtain required proof.

We have well-known theorem presented by Steprāns Juris in [11] about a group G, which is a discretely normed abelian group. Therefore, we have following corollaries.

Corollary 8. For free abelian group $G$, a function $\chi$ is a solution of Equation (9) if and only if there exists an additive function $\alpha: G \rightarrow \mathbb{R}$, such that $\chi(x)=e^{|\alpha(x)|}$ for any $x \in G \backslash\{1\}$.

Corollary 9. Suppose that $G$ is a free abelian group, then $\chi$ is a solution of functional Equation (10) satisfying (11) if and only if there exists an additive function $\alpha: G \rightarrow \mathbb{R}$, such that $\chi(x)=e^{|\alpha(x)|}$ for any $x \in G \backslash\{1\}$.

Theorem 7. Let $G$ be any group and let a function $\chi: G \rightarrow \mathbb{R}$ is a solution of Equation (10) and (11), which is not identically zero, then there exists a normal subgroup $H_{\chi}=\left\{v \in G \mid \chi\left(v^{2}\right)=1\right\}$ of $G$.

Proof. Because the function $\chi: G \rightarrow \mathbb{R}$ satisfying the Equations (10) and (11), then by Theorem 5 , we can obtain that

$$
\max \left\{\chi(x y), \chi\left(x y^{-1}\right)\right\}=\chi(x) \chi(y) \quad \text { for all } x, y \in G .
$$

For neutral element 1 , we can write $\chi\left(1^{2}\right)=\chi(1)=1$, then $1 \in H_{\chi}$. Let $v \in H_{\chi}$, then $\chi\left(v^{2}\right)=1$. From Equation (13), follows immediately that $\chi$ is even; therefore, $\chi\left(v^{-2}\right)=\chi\left(v^{2}\right)=1$, hence $v^{-1} \in$ $H_{\chi}$. Let $u, v \in H_{\chi}$, therefore, by property of $H_{\chi}, \chi\left(u^{2}\right)=1$ and $\chi\left(v^{2}\right)=1$. We can deduce from maximum Equation (13) that

$$
\begin{aligned}
\chi\left(u^{2} v^{2}\right) & =\chi\left(u^{2} v^{2}\right) \chi\left(v^{2}\right) \\
& =\max \left\{\chi\left(u^{2} v^{4}\right), \chi\left(u^{2}\right)\right\} \\
& \geq \chi\left(u^{2}\right) \\
\chi\left(u^{2} v^{2}\right) & \geq \chi\left(u^{2}\right) \chi\left(v^{2}\right) . \\
\chi\left(u^{2}\right) \chi\left(v^{2}\right) & =\max \left\{\chi\left(u^{2} v^{2}\right), \chi\left(u^{2} v^{-2}\right)\right\} \\
& \geq \chi\left(u^{2} v^{2}\right) \\
\chi\left(u^{2}\right) \chi\left(v^{2}\right) & \geq \chi\left(u^{2} v^{2}\right) .
\end{aligned}
$$

From inequalities (14) and (15), we can calculate $\chi\left(u^{2} v^{2}\right)=\chi\left(u^{2}\right) \chi\left(v^{2}\right)=1$, which implies that $u v \in H_{\chi}$. Therefore, $H_{\chi}$ is a subroup of $G$. Additionally, Equation (13) yields that $\chi$ is central, therefore $\chi(x v)=\chi(v x)$, for every $x \in G$ and $v \in H_{\chi}$. Hence, $H_{\chi}$ is a normal subgroup of $G$.

Corollary 10. For any group $G$, let a function $\chi$ on group $G$ satisfying (13), which is not identically zero, then $\chi: G / H_{\chi} \rightarrow \mathbb{R}$ also satisfies

$$
\chi(x v)=\chi(x) \chi(v) \quad \text { for any } x \in G, v \in H_{\chi} .
$$


Moreover,

$$
\chi\left(x v^{2}\right)=\chi\left(v^{2} x\right)=\chi(x) \quad \text { for any } x \in G, v \in H_{\chi}
$$

Proof. Let $x \in G$ be an arbitrary and assume that $v \in H_{\chi}$, then $\chi\left(v^{2}\right)=1$. We can deduce from (13) that

$$
\chi(x v) \chi(v)=\max \left\{\chi\left(x v^{2}\right), \chi(x)\right\} \geq \chi(x) \chi\left(v^{2}\right),
$$

which provides that $\chi(x v) \chi(v) \geq \chi(x) \chi\left(v^{2}\right)$. Applying condition (11), we compute $\chi(x v) \geq \chi(x) \chi(v)$. Additionally, from (13), we have $\chi(x) \chi(v) \geq \chi(x v)$, so we can evaluate $\chi(x v)=\chi(x) \chi(v)$ for any $x \in G$ and $v \in H_{\chi}$.

Because $H_{\chi}$ is a subgroup of $G$, then $v^{2} \in H_{\chi}$, so we can see that $\chi\left(x v^{2}\right)=\chi(x) \chi\left(v^{2}\right)$ for any $x \in G$ and $v^{2} \in H_{\chi}$, therefore $\chi\left(x v^{2}\right)=\chi(x)$. Writing $x$ instead of $y$ and $v$ instead of $x$ in (13), we can conclude $\chi\left(x^{-1} v x\right)=\chi(v)$ for any $x \in G$, then writing $x v^{2}$ instead of $v$, we have $\chi\left(v^{2} x\right)=\chi\left(x v^{2}\right)=\chi(x)$.

Corollary 11. Let $G$ be a group and function $\chi: G \rightarrow \mathbb{R}$ satisfies the Kannappan condition. If the maximum functional Equation (13) holds, then $\min \left\{\chi(x y), \chi\left(x y^{-1}\right)\right\} \in H_{\chi}$ for any $x, y \in G$.

Proof. Assume that $\chi$ satisfies the Equation (9) and Kannappan condition. Subsequently, from Theorem 5, we can conclude that

$$
\min \left\{\chi(x y), \chi\left(x y^{-1}\right)\right\}=\chi(x) \chi(y)^{-1}, \quad(\text { when } \chi(x) \geq \chi(y))
$$

or

$$
\min \left\{\chi(x y), \chi\left(x y^{-1}\right)\right\}=\left[\chi(x) \chi(y)^{-1}\right]^{-1}, \quad(\text { when } \chi(x)<\chi(y))
$$

combining both cases, we can see that $\min \left\{\chi(x y), \chi\left(x y^{-1}\right)\right\}=\left[\min \left\{\chi(x y), \chi\left(x y^{-1}\right)\right\}\right]^{-1}$, therefore $\min \left\{\chi(x y), \chi\left(x y^{-1}\right)\right\}^{2}=1$. In either case, it follows that $\min \left\{\chi(x y), \chi\left(x y^{-1}\right)\right\} \in H_{\chi}$ for every $x, y \in G$.

\section{Stability of Maximum Functional Equation (1)}

In order to check the stability of MFE (1) in two-variables $x$ and $y$, at the first stage, we put $y=x$ in (1) and derive the stability of MFE (1) in a single variable, as follows:

Theorem 8. Let $G$ be any group and a function $\eta: G \rightarrow \mathbb{R}$ satisfies

$$
\left|\max \left\{\eta\left(x^{2}\right), \eta(1)\right\}-2 \eta(x)\right| \leq \lambda \quad \text { for any } x \in G,
$$

for some $\lambda \geq 0$. Subsequently, we can obtain a solution $\chi: G \rightarrow \mathbb{R}$ of

$$
\max \left\{\chi\left(x^{2}\right), \chi(1)\right\}=2 \chi(x) \quad \text { for any } x \in G
$$

such that

$$
-3 \lambda \leq \chi(x)-\eta(x) \leq \lambda \quad \text { for any } x \in G .
$$

Additionally, $\chi$ is given by

$$
\chi(x)=\lim _{n \rightarrow \infty} \frac{1}{2^{n}} \eta\left(x^{2^{n}}\right) \quad x \in G .
$$

Additionally, by (18), $\chi$ is uniquely determined, and by (19), the requirement of $\chi-\eta$ to be bounded is also satisfied. 
Proof. First, put $x=1$ in (17), then we have $|\max \{\eta(1), \eta(1)\}-2 \eta(1)| \leq \lambda$, which implies that $|\eta(1)| \leq \lambda$. Furthermore, (17) also implies that

$$
\begin{aligned}
-\lambda+2 \eta(x) & \leq \max \left\{\eta\left(x^{2}\right), \eta(1)\right\} \leq \lambda+2 \eta(x) \\
-\lambda & \leq \eta(1) \leq \lambda+2 \eta(x) \\
-\lambda & \leq \eta(x) \quad \text { for any } x \in G .
\end{aligned}
$$

Writing $x^{2}$ instead of $x$, then also $-\lambda \leq \eta\left(x^{2}\right)$, so we can get that

$$
\begin{aligned}
& \eta(1) \leq \lambda=2 \lambda-\lambda \leq 2 \lambda+\eta\left(x^{2}\right) \\
& \eta(1) \leq 2 \lambda+\eta\left(x^{2}\right) \quad \text { for any } x \in G .
\end{aligned}
$$

From (17), we have

$$
\begin{array}{ll} 
& 2 \eta(x) \leq \lambda+\max \left\{\eta\left(x^{2}\right), \eta(1)\right\} \\
& 2 \eta(x) \leq \lambda+\eta(1) \leq 3 \lambda+\eta\left(x^{2}\right) \quad \text { (using (22)) } \\
\text { or } \quad 2 \eta(x) \leq \lambda+\eta\left(x^{2}\right) \leq 3 \lambda+\eta\left(x^{2}\right) .
\end{array}
$$

Combining both cases, we can conclude

$$
-3 \lambda \leq \eta\left(x^{2}\right)-2 \eta(x)
$$

From (17), we have $\max \left\{\eta\left(x^{2}\right), \eta(1)\right\} \leq \lambda+2 \eta(x)$, which is only possible when

$$
\eta\left(x^{2}\right)-2 \eta(x) \leq \lambda
$$

From inequalities (23) and (24), we have

$$
-3 \lambda \leq \eta\left(x^{2}\right)-2 \eta(x) \leq \lambda \quad \text { for all } x \in G .
$$

When we observe (25), it can be seen that the function $\chi: G \rightarrow \mathbb{R}$ presented in (20) exists and this $\chi$ satisfies

$$
\chi\left(x^{2}\right)=2 \chi(x) \quad \text { for all } x \in G,
$$

also $\chi$ fulfills (19). Furthermore, writing $x^{2^{n}}$ instead of $x$ in (21), dividing by $2^{n}$ and taking a limit $n \rightarrow \infty$ and, using (20), we can obtain that $\chi(x) \geq 0$ for any $x \in G$. Hence we can obtain (18) from (26). Furthermore, when we consider (18), then we can easily see the uniqueness of $\chi$, due to the result that $\chi\left(x^{2}\right)=2 \chi(x) \geq 0$ for any $x \in G$.

By utilizing Theorem (8), we are going to derive the stability of Equation (1) in two-variables $x$ and $y$.

Theorem 9. Let $G$ be any group and function $\eta: G \rightarrow \mathbb{R}$ satisfies

$$
\left|\max \left\{\eta(x y), \eta\left(x y^{-1}\right)\right\}-\eta(x)-\eta(y)\right| \leq \lambda \quad \text { for any } x, y \in G,
$$

for some $\lambda \geq 0$. Subsequently, we can evaluate a unique solution $\chi: G \rightarrow \mathbb{R}$ of

$$
\max \left\{\chi(x y), \chi\left(x y^{-1}\right)\right\}=\chi(x)+\chi(y) \quad \text { for any } x, y \in G
$$


such that

$$
-3 \lambda \leq \chi(x)-\eta(x) \leq \lambda \quad \text { for any } x \in G
$$

Additionally,

$$
\chi(x)=\lim _{n \rightarrow \infty} \frac{1}{2^{n}} \eta\left(x^{2^{n}}\right) \quad \text { for any } x \in G .
$$

Proof. Applying the Theorem (8), we can easily prove the required results. First, writing $x$ instead of $y$ in (27), then, from Theorem (8), we can obtain a function $\chi: G \rightarrow \mathbb{R}$. We also need to prove that this function $\chi$ satisfies (28). To obtain the required maximum functional Equation (28), we need to write $x^{2^{n}}$ instead of $x$ and $y^{2^{n}}$ instead of $y$ in (27), dividing inequality by $2^{n}$ and, applying limit $n \rightarrow \infty$ and also using Equation (30).

Theorem 10. Let $G$ be a group and function $\eta: G \rightarrow \mathbb{R}$ fulfills (27), then, for any $x, y \in G$, it holds the condition

$$
\lim _{n \rightarrow \infty} \frac{1}{2^{n}}\left[\max \left\{\eta\left(x^{2^{n}} y^{2^{n}}\right), \eta\left(x^{2^{n}} y^{-2^{n}}\right)\right\}-\max \left\{\eta\left([x y]^{2^{n}}\right), \eta\left(\left[x y^{-1}\right]^{2^{n}}\right)\right\}\right]=0
$$

if and only if the Equation (28) is satisfied.

Proof. Assume that maximum Equation (28) is satisfied. For every $x, y \in G$, we have

$$
\begin{aligned}
\mid \max \left\{\eta\left(x^{2^{n}} y^{2^{n}}\right), \eta\left(x^{2^{n}} y^{-2^{n}}\right)\right\}- & \max \left\{\eta\left([x y]^{2^{n}}\right), \eta\left(\left[x y^{-1}\right]^{2^{n}}\right)\right\} \mid \\
& \leq\left|\max \left\{\eta\left(x^{2^{n}} y^{2^{n}}\right), \eta\left(x^{2^{n}} y^{-2^{n}}\right)\right\}-\eta\left(x^{2^{n}}\right)-\eta\left(y^{2^{n}}\right)\right| \\
& +\left|\max \left\{\eta\left([x y]^{2^{n}}\right), \eta\left(\left[x y^{-1}\right]^{2^{n}}\right)\right\}-\eta\left(x^{2^{n}}\right)-\eta\left(y^{2^{n}}\right)\right| \\
& \leq \lambda+\left|\max \left\{\eta\left([x y]^{2^{n}}\right), \eta\left(\left[x y^{-1}\right]^{2^{n}}\right)\right\}-\eta\left(x^{2^{n}}\right)-\eta\left(y^{2^{n}}\right)\right| .
\end{aligned}
$$

To obtain the required result, first, we divide both sides by $2^{n}$, apply limit as $n \rightarrow$ $\infty$ and utilize the given condition (28), we obtain that, for any $x, y \in G$, we can get $\lim _{n \rightarrow \infty} \frac{1}{2^{n}}\left[\max \left\{\eta\left(x^{2^{n}} y^{2^{n}}\right), \eta\left(x^{2^{n}} y^{-2^{n}}\right)\right\}-\max \left\{\eta\left([x y]^{2^{n}}\right), \eta\left(\left[x y^{-1}\right]^{2^{n}}\right)\right\}\right]=0$.

Conversely, assume that condition (31) holds. Putting $x^{2^{n}}$ and $y^{2^{n}}$ in (27) instead of $x$ and $y$, respectively, then dividing by $2^{n}$ and taking limit $n \rightarrow \infty$, we can compute

$$
\begin{gathered}
\lim _{n \rightarrow \infty} \frac{1}{2^{n}}\left[\max \left\{\eta\left(x^{2^{n}} y^{2^{n}}\right), \eta\left(x^{2^{n}} y^{-2^{n}}\right)\right\}=\chi(x)+\chi(y),\right. \\
\lim _{n \rightarrow \infty} \frac{1}{2^{n}} \max \left\{\eta\left([x y]^{2^{n}}\right), \eta\left(\left[x y^{-1}\right]^{2^{n}}\right)\right\}=\max \left\{\chi(x y), \chi\left(x y^{-1}\right)\right\} .
\end{gathered}
$$

Given condition (31), yielding that $\max \left\{\chi(x y), \chi\left(x y^{-1}\right)\right\}=\chi(x)+\chi(y)$.

Furthermore, the given condition (31) for function $\eta$ is not directly associated to the properties of a group $G$, but we can see some useful results regarding group $G$. The resulting condition presented below is equivalent to the above condition (31), as there exists a subsequence of $\mathbb{N}$, such that

$$
\lim _{n \rightarrow \infty} \frac{1}{2^{m(n)}}\left[\max \left\{\eta\left(x^{2^{m(n)}} y^{2^{m(n)}}\right), \eta\left(x^{2^{m(n)}} y^{-2^{m(n)}}\right)\right\}-\max \left\{\eta\left([x y]^{2^{m(n)}}\right), \eta\left(\left[x y^{-1}\right]^{2^{m(n)}}\right)\right\}\right]=0 .
$$

This refers to the function $\eta$ and completely satisfactory, due to the fact that both limits

$$
\lim _{n \rightarrow \infty} \frac{1}{2^{m(n)}} \max \left\{\eta\left(x^{2^{m(n)}} y^{2^{m(n)}}\right), \eta\left(x^{2^{m(n)}} y^{-2^{m(n)}}\right)\right\}
$$


and

$$
\lim _{n \rightarrow \infty} \frac{1}{2^{m(n)}} \max \left\{\eta\left([x y]^{2^{m(n)}}\right), \eta\left(\left[x y^{-1}\right]^{2^{m(n)}}\right)\right\}
$$

are finite and exists.

Corollary 12. A function $\eta: G \rightarrow \mathbb{R}$ fulfills (18), then it satisfies the condition

$$
\lim _{n \rightarrow \infty} \frac{1}{2^{n}}\left[\max \left\{\eta\left(x^{2^{n}} y^{2^{n}}\right), \eta\left(x^{2^{n}} y^{-2^{n}}\right)\right\}-\eta\left([x y]^{2^{n}}\right)\right]=0 \quad \text { for all } x, y \in G
$$

if and only if $\chi(x y)=\chi(x)+\chi(y)$ for any $x, y \in G$.

Remark 1. (i) The given condition (31) holds when group $G$ is an $n-$ Abelian group (for any integer $n$, a group $G$ is called an $n$-Abelian group if $(a b)^{n}=a^{n} b^{n}$, for any $a, b \in G$, see $[12,13]$ ).

(ii) The condition (31) also satisfies when group $G$ belongs to the class $C_{n}$ for any natural number $n \in \mathbb{N}$ (for all $n \in \mathbb{N}, C_{n}$ is denoted as the class of groups, which satisfies the relation $b^{n} a^{n}=a^{n} b^{n}$ ).

(iii) When $\chi$ is central, then condition (31) also holds.

Theorem 11. If maximum functional Equation (1) is stable on group $G$, then every free abelian group $K$ can be embedded into a group $G$.

Proof. Assume that $\eta: G \rightarrow \mathbb{R}$ and let $\lambda \geq 0$ be such that

$$
\left|\max \left\{\eta(x y), \eta\left(x y^{-1}\right)\right\}-\eta(x)-\eta(y)\right| \leq \lambda \quad \text { for any } x, y \in G .
$$

Because every free abelian group is a torsion-free, $K$ is torsion-free group. Subsequently, from HNN-extensions, any torsion-free group $K$ can be embedded into a group $G$, if for all $k \in K$, there exists $g \in G$ such that $\mathrm{gkg}^{-1}=k^{2}[14,15]$. Assume that $K$ is embedded into a group $G$. Subsequently, inequality (33) can be rewritten as

$$
\left|\max \left\{\eta(k g), \eta\left(k g^{-1}\right)\right\}-\eta(k)-\eta(g)\right| \leq \lambda \quad \text { for any } k, g \in G .
$$

We need to show that $\eta$ is bounded. The result is obvious for $\lambda=0$, so let $\lambda$ be positive. More specifically, we prove that $|\eta(k)|<2 \lambda$ for all $k \in G$. Afterwards, from (34), we have two possibilities, either $\left|\eta\left(k^{-1}\right)-\eta(k)-\eta(g)\right| \leq \lambda$ or $|\eta(k g)-\eta(k)-\eta(g)| \leq \lambda$. Let us consider first possibility and put $k=g=1$, then we can conclude that $|\eta(1)| \leq \lambda$. Put $g=k$, then we have

$$
\begin{aligned}
|\eta(1)-2 \eta(k)| & \leq \lambda \\
|2 \eta(k)|-|\eta(1)| & \leq \lambda \\
2|\eta(k)| & \leq \lambda+|\eta(1)| \\
2|\eta(k)| & \leq \lambda+\lambda \\
|\eta(k)| & \leq \lambda .
\end{aligned}
$$

For second possibilty, we have

$$
|\eta(k g)-\eta(k)-\eta(g)| \leq \lambda
$$


On the contrary, let $|\eta(k)| \geq 2 \lambda$ for some arbitrary $k \in G$. Put $g=k$ in (35), then we have

$$
\begin{aligned}
\left|\eta\left(k^{2}\right)-2 \eta(k)\right| & \leq \lambda \\
\left|2 \eta(k)-\eta\left(k^{2}\right)\right| & \leq \lambda \\
2|\eta(k)|-\lambda & \leq\left|\eta\left(k^{2}\right)\right| \\
3 \lambda & \leq\left|\eta\left(k^{2}\right)\right| .
\end{aligned}
$$

Again, put $g=k^{2}$ in (35), then

$$
\begin{aligned}
\left|\eta\left(k^{3}\right)-\eta(k)-\eta\left(k^{2}\right)\right| & \leq \lambda \\
\left|\eta(k)+\eta\left(k^{2}\right)-\eta\left(k^{3}\right)\right| & \leq \lambda \\
|\eta(k)|+\left|\eta\left(k^{2}\right)\right| & \leq \lambda+\left|\eta\left(k^{3}\right)\right| \\
5 \lambda-\lambda & \leq\left|\eta\left(k^{3}\right)\right| \\
4 \lambda & \leq\left|\eta\left(k^{3}\right)\right| .
\end{aligned}
$$

Repeating again, for $g=k^{3}$, we can determine $\left|\eta\left(k^{4}\right)\right| \geq 4 \lambda$. Continuing this process, we can evaluate that

$$
(m+1) \lambda \leq\left|\eta\left(k^{m}\right)\right|, \quad \text { for } \mathrm{m}=1,2, \cdots,
$$

which determines that when $m$ varies, then $\eta\left(k^{m}\right)$ is unbounded.

Furthermore, pick an arbitrary element $g \in G$, such that $k^{2}=g \mathrm{~kg}^{-1}$. Subsequently, for every integer $m>0$, we have $k^{2 m}=g k^{m} g^{-1}$. Therefore, for every $m$, put $g=k^{m}$ and $k=k^{m}$ in (35), then

$$
\begin{aligned}
\left|\eta\left(k^{2 m}\right)-2 \eta\left(k^{m}\right)\right| & \leq \lambda \\
\left|\eta\left(g k^{m} g^{-1}\right)-2 \eta\left(k^{m}\right)\right| & \leq \lambda .
\end{aligned}
$$

Additionally, inequality (35) follows that

$$
\left|\eta\left(g k^{m} g^{-1}\right)-\eta(g)-\eta\left(k^{m} g^{-1}\right)\right| \leq \lambda \quad \text { and } \quad\left|\eta\left(k^{m} g^{-1}\right)-\eta\left(k^{m}\right)-\eta\left(g^{-1}\right)\right| \leq \lambda,
$$

thus, we can conclude that

$$
\begin{aligned}
\left|\eta\left(g k^{m} g^{-1}\right)-\eta(g)-\eta\left(k^{m}\right)-\eta\left(g^{-1}\right)\right| & \leq\left|\eta\left(g k^{m} g^{-1}\right)-\eta(g)-\eta\left(k^{m} g^{-1}\right)\right| \\
& +\left|\eta\left(k^{m} g^{-1}\right)-\eta\left(k^{m}\right)-\eta\left(g^{-1}\right)\right| \\
\left|\eta\left(g k^{m} g^{-1}\right)-\eta(g)-\eta\left(k^{m}\right)-\eta\left(g^{-1}\right)\right| & \leq 2 \lambda
\end{aligned}
$$

From (36) and (37), we have

$$
\begin{aligned}
\left|\eta\left(g k^{m} g^{-1}\right)-2 \eta\left(k^{m}\right)+\eta\left(k^{m}\right)\right| & \leq 2 \lambda+|\eta(g)|+\left|\eta\left(g^{-1}\right)\right| \\
\left|\eta\left(k^{m}\right)\right| & \leq 2 \lambda+|\eta(g)|+\left|\eta\left(g^{-1}\right)\right|+\left|\eta\left(g k^{m} g^{-1}\right)-2 \eta\left(k^{m}\right)\right| \\
\left|\eta\left(k^{m}\right)\right| & \leq 5 \lambda \quad \text { for } \mathrm{m}=1,2, \cdots,
\end{aligned}
$$

which gives a contradiction. Hence, $\eta$ is bounded, which completes the required proof.

Corollary 13. If maximum functional Equation (1) is stable on group $G$, then any discretely normed abelian group $K$ can be embedded into a group $G$.

Proof. Because $K$ is a discretely normed abelian group, then, by applying theorem from [11], $K$ is a free group; hence it can be embedded into a group $G$. 
Theorem 12. A function $\eta: G \rightarrow \mathbb{R}$ satisfying (27) is bounded on the commutator group $G_{1}$ of the subgroup $G^{\prime}$ of $G$ if the condition (31) is satisfied.

Proof. Assume that condition (31) is satisfied. Subsequently, by Theorem 10, we can get that $\chi(x)+$ $\chi(y)=\max \left\{\chi(x y), \chi\left(x y^{-1}\right)\right\}$ holds for any $x, y \in G$. Because this maximum equation holds; therefore, by Theorem 1, there exists an additive function $\alpha: G \rightarrow \mathbb{R}$, such that $\chi(x)=|\alpha(x)|$ for any $x \in G$. For $a, b \in G$, take $a^{-1} b^{-1} a b \in G$, then

$$
\begin{aligned}
\chi\left(a^{-1} b^{-1} a b\right) & =\left|\alpha\left(a^{-1} b^{-1} a b\right)\right|=\left|\alpha\left(a^{-1}\right)+\alpha\left(b^{-1}\right)+\alpha(a)+\alpha(b)\right| \\
& =\left|\alpha\left(a^{-1} a\right)+\alpha\left(b^{-1} b\right)\right|=|\alpha(1)+\alpha(1)|=|0+0| \\
\chi\left(a^{-1} b^{-1} a b\right) & =0 .
\end{aligned}
$$

Because $\chi$ is zero on the commutator group $G_{1}$ of the subgroup $G^{\prime}$ of $G, \eta$ is bounded on commutator group $G_{1}$.

Corollary 14. If a function $\eta: G \rightarrow \mathbb{R}$ satisfies the condition

$$
\lim _{n \rightarrow \infty} \frac{1}{2^{n}}\left[\max \left\{\eta\left(x^{2^{n}} y^{2^{n}}\right), \eta\left(x^{2^{n}} y^{-2^{n}}\right)\right\}-\eta\left([x y]^{2^{n}}\right)\right]=0 \quad \text { for any } x, y \in G
$$

then $\eta$ is bounded on the commutator group $G_{1}$.

Author Contributions: Writing—original draft, M.S., Writing—review and editing, Q.L. and Y.L. All authors have read and agreed to the published version of the manuscript.

Funding: This research was funded by National Natural Science Foundation of China grant number 11971493.

Acknowledgments: The authors thank anonymous referees for their remarkable comments, suggestions, and ideas that help to improve this paper.

Conflicts of Interest: The authors declare no conflict of interest.

\section{References}

1. Volkmann, P. Charakterisierung des Betrages reellwertiger additiver Funktionen auf Gruppen. KITopen 2017, 4, 99191049.

2. Toborg, I. On the functional equation $f(x)+f(y)=\max \left\{f(x y), f\left(x y^{-1}\right)\right\}$ on groups. Arch. Math. 2017, 109, 215-221. [CrossRef]

3. Gilanyi, A.; Nagatou, K.; Volkmann, P. Stability of a functional equation coming from the characterization of the absolute value of additive functions. Ann. Funct. Anal. 2010, 1, 1-6. [CrossRef]

4. Kochanek, T. On a composite functional equation fulfilled by modulus of an additive function. Aequ. Math. 2010, 80, 155-172. [CrossRef]

5. Chaljub-Simon, A.; Volkmann, P. Caractérisation du module d'une fonction additive à l'aide d'une équation fonctionnelle. Aequ. Math 1994, 47, 60-68. [CrossRef]

6. Jarczyk, W.; Volkmann, P. On functional equations in connection with the absolute value of additive functions. Ser. Math. Catoviciensis Debr. 2010, 32, 11.

7. Badora, R.; Przebieracz, B.; Volkmann, P. On Tabor groupoids and stability of some functional equations. Aequ. Math 2014, 87, 165-171. [CrossRef]

8. Przebieracz, B. Superstability of some functional equation. Ser. Math. Catoviciensis Debr. 2010, 31, 4.

9. Przebieracz, B. The stability of functional equation $\min \{f(x+y), f(x-y)\}=|f(x)-f(y)|$. J. Inequal. Appl. 2011, 1, 1-6. [CrossRef]

10. Kannappan, Pl. The functional equation $f(x y)+f\left(x y^{-1}\right)=2 f(x) f(y)$ for groups. Proc. Am. Math. Soc. $1968,19,69-74$.

11. Steprāns, J. A characterization of free abelian groups. Proc. Am. Math. Soc. 1985, 93, 347-349. [CrossRef]

12. Alperin, J.L. A classification of $n$-abelian groups. Can. J. Math. 1969, 21, 1238-1244. [CrossRef]

13. Baer, R. Factorization of $n$-soluble and $n$-nilpotent groups. Proc. Am. Math. Soc. 1953, 4, 15-26. 
14. Higman, G.; Neumann, B.H.; Neuman, H. Embedding theorems for groups. J. Lond. Math. Soc. 1949, 1, 247-254. [CrossRef]

15. Magnus, W.; Karrass, A.; Solitar, D. Combinatorial Group Theory: Presentations of Groups in Terms of Generators and Relations; Courier Corporation: North Chelmsford, MA, USA, 2004.

Publisher's Note: MDPI stays neutral with regard to jurisdictional claims in published maps and institutional affiliations.

(C) 2020 by the authors. Licensee MDPI, Basel, Switzerland. This article is an open access article distributed under the terms and conditions of the Creative Commons Attribution (CC BY) license (http:/ / creativecommons.org/licenses/by/4.0/). 